\title{
Wie relevant sind alternative Behandlungsstrategien neben der Kognitiven Verhaltenstherapie?
}

\author{
Ulrike Ehlert \\ Klinische Psychologie und Psychotherapie, Universität Zürich, Schweiz
}

Leserinnen und Leser der Zeitschrift VERHALTENSTHERAPIE wenden Verhaltenstherapie als professionelle Therapeuten und Therapeutinnen an und wollen wissen, welche neuen Erkenntnisse im Bereich der Verhaltenstherapie vorliegen. Diese Aussage impliziert, dass es ein breites Spektrum von Anwendungsfeldern für Verhaltenstherapie gibt, ein großes Repertoire an Methoden vorhanden ist und fortlaufend empirische Forschung durchgeführt wird [vgl. Linden, 2007].

Die Anwendung verschiedener verhaltenstherapeutischer Methoden kann sowohl unter dem Aspekt der Störungsspezifität als auch im Kontext des jeweiligen Settings betrachtet werden. Zur kognitiven Verhaltenstherapie (KVT) existieren inzwischen viele ausgezeichnete randomisierte Therapiestudien, die die Wirksamkeit des Verfahrens geprüft haben. Publikationsdatenbanken wie PubMed (www.ncbi.nlm.nih.gov/) oder die metaanalytischen Auswertungen durch die Cochrane Collaboration (www.cochrane.org/) geben einen Überblick über die beeindruckende Anwendungsbreite der KVT. In den entsprechenden Wirksamkeitsstudien werden nicht nur Wartegruppendesigns oder Vergleiche zwischen KVT und «Treatment as usual» durchgeführt, sondern differenzierte Studiendesigns eingesetzt, mittels derer KVT, psychopharmakologische Behandlungen und die Kombination beider Behandlungsformen miteinander verglichen werden. Auch liegen einige Therapievergleichsstudien zwischen KVT und anderen Therapieformen wie beispielsweise interpersonaler Therapie vor. Aus einer Fülle von Untersuchungen geht hervor, dass KVT ein wirksames Therapieverfahren ist. Dies gilt sowohl unter hoch standardisierten Behandlungsbedingungen als auch in praktischen Settings wie beispielsweise der ambulanten Patientenversorgung. Insbesondere für den Bereich der Angststörungen liegen inzwischen nicht nur die erwähnten Wirksamkeitsstudien, sondern auch Langzeitkatamnesen vor, die die Nachhaltigkeit der Therapieeffekte nachweisen.

Wunderbar! Wir können also mit großer Zufriedenheit unser therapeutisches Wissen und unsere praktisch-therapeutischen Kompetenzen einsetzen und davon überzeugt sein, dass «unsere» Therapieangebote an Patientinnen und Patienten wirksam sind.

Ganz so einfach scheint es allerdings doch nicht zu sein. Für bestimmte Störungsbilder wie beispielsweise die BorderlinePersönlichkeitsstörung liegen entsprechend der CochraneAnalysen noch nicht genügend Studien vor; daher ist eine umfassende Einschätzung der Wirksamkeit dialektisch-behavioraler Therapie (DBT) noch nicht möglich [Binks et al., 2006]. Für bestimmte andere Formen von Persönlichkeitsstörungen beginnen Forschergruppen erst, Behandlungskonzepte auf verhaltenstherapeutischer Basis zu entwickeln und erste Evaluationen vorzunehmen [z.B. Zorn et al., 2007]. Für die affektiven Störungen gibt es inzwischen zwar viele Publikationen, die eine gute Wirksamkeit der KVT bei leichten bis mittelschweren depressiven Störungen bei unterschiedlichen Altersgruppen belegen [z.B. Laidlaw et al., 2008]; jedoch mehren sich die Einschätzungen, dass bei schwerwiegenden und chronifizierten affektiven Störungen eine Kombination aus antidepressiver Medikation und KVT die wirksamste Therapiestrategie ist [Durham et al., 2005; Hautzinger, 2008; Hautzinger und Meyer, 2007].

Bei schweren, komplexen und/oder komorbiden psychischen Störungen bleiben Therapieerfolge häufig aus. Dies gilt nicht nur für psychotherapeutische Interventionen, sondern auch für pharmakologische Behandlungen bzw. deren Kombination. Am Beispiel depressiver Störungen zeigt sich sehr deutlich, dass für derart schwerkranke Menschen alternative Behandlungsstrategien in Erwägung gezogen werden müssen. Üblicherweise fällt die Entscheidung, alternative Behandlungsmethoden einzusetzen, interdisziplinär und erst nach Ausschöpfung der oben genannten Methoden. Unter reiflicher Abwägung aller Risiken und Vorteile für den Patienten können beispielsweise lang erprobte Therapieverfahren wie

\begin{tabular}{ll}
\hline KARGER & (c) 2008 S. Karger GmbH, Freiburg \\
Fax +497614520714 & Accessible online at: \\
$\begin{array}{l}\text { E-mail Information@Karger.de } \\
\text { www.karger.com }\end{array}$ & www.karger.com/ver \\
&
\end{tabular}


die Elektrokrampftherapie eingesetzt werden. Basierend auf neurobiologischen Überlegungen zur Dysregulation spezifischer Neurotransmittersysteme (z.B. Serotonin- oder Dopaminstoffwechsel) werden in der aktuellen Literatur neue, derzeit noch experimentelle Untersuchungsansätze beschrieben. Dazu gehören die repeditive transkranielle Magnetstimulation, die Vagusnervstimulation [vgl. Nemeroff et al., 2006] oder die chronische Tiefenstimulation spezifischer Hirnareale nach operativer Sondenimplantation (Deep brain stimulation, DBS) [Schlaepfer et al., 2008]. Im Interview auf Seite 120 dieser Ausgabe der Verhaltenstherapie stellt Helen Mayberg von der Emory University in Atlanta (USA) dieses Verfahren und ihre künftigen Forschungsabsichten vor [vgl. auch Mayberg et al., 2005].

Ich denke nicht, dass die Zukunft der Behandlung psychischer Störungen im Einsatz neurobiologischer Therapieverfahren liegt. Als Psychotherapeuten sollten wir diese neuen Verfahren jedoch kennen, über ihre Risiken Bescheid wissen, aber auch die potenziellen Therapiechancen für schwerstkranke Patientinnen und Patienten mit chronifizierten Störungen anerkennen.

\section{Literatur}

Binks CA, Fenton M, McCarthy L, Lee T, Adams CE, Duggan C: Psychological therapies for people with borderline personality disorder. Cochrane Database Systc Rev 2006;CD005652.

Durham RC, Chambers JA, Power KG, Sharp DM, Macdonald RR, Major KA, Dow MG, Gumley AI: Long-term outcome of cognitive behaviour therapy clinical trials in central Scotland. Health Technol Assess 2005;9:1-174.

Hautzinger M: Psychotherapie der Depression. Bundesgesundheitsbl Gesundheitsforsch Gesundheitsschutz 2008;20:Epub ahead of print.

Hautzinger M, Meyer TD: Psychotherapie bei bipolaren affektiven Störungen. Nervenarzt 2007;78: 1248-60.

Laidlaw K, Davidson K, Toner H, Jackson G, Clark S, Law J, Howley M, Bowie G, Connery H, Cross S: A randomised controlled trial of cognitive behaviour therapy vs treatment as usual in the treatment of mild to moderate late life depression. Int $\mathrm{J}$ Geriatr Psychiatry 2008;Epub ahead of print.

Linden M: Aaron T. Beck: «Störungen der korrekten Informationsverarbeitung führen zu Stimmungsproblemen». Verhaltenstherapie 2007;17:195-196.

Mayberg HS, Lozano AM, Voon V, McNeely HE, Seminowicz D, Hamani C, Schwalb JM, Kennedy SH: Deep brain stimulation for treatment-resistant depression. Neuron 2005;45:651-60.

Nemeroff CB, Mayberg HS, Krahl SE, McNamara J, Frazer A, Henry TR, George MS, Charney DS, Brannan SK: VNS therapy in treatment-resistant depression: clinical evidence and putative neurobiological mechanisms. Neuropsychopharmacology 2006; 31:1345-1355.

Schlaepfer TE, Cohen MX, Frick C, Kosel M, Brodesser D, Axmacher N, Joe AY, Kreft M, Lenartz D, Sturm V: Deep brain stimulation to reward circuitry alleviates anhedonia in refractory major depression. Neuropsychopharmacology 2008;33: 368-377.

Zorn P, Roder V, Müller DR, Tschacher W, Thommen M: Schemazentrierte emotivbehaviorale Therapie (SET): Eine randomisierte Evaluationsstudie an Patienten mit Persönlichkeitsstörungen aus den Clustern B und C. Verhaltenstherapie 2007;17: $233-241$. 